\title{
Comunicación asertiva y su relación enfermero-paciente por el uso constante de la mascarilla
}

Erika Paola Yanchapanta Analuisa

eyanchapanta5231@uta.edu.ec https://orcid.org/0000-0001-8621-9334 Universidad Técnica de Ambato Ambato-Ecuador

Eulalia Isabel Analuisa Jiménez

Docente de la Carrera De Enfermería ei.analuisa@uta.edu.ec https://orcid.org/0000-0002-7906-7640

\section{RESUMEN}

Universidad Técnica de Ambato, Ecuador

Introducción: La comunicación asertiva en el entorno es un proceso fundamental en toda relación social, que regula y hace posible la interacción con las personas a la hora de prestar cuidados integrales y de calidad, mediante la utilización de terminologías claras y comprensibles, permitiendo así ejercer un vínculo de confianza y seguridad entre enfermero-paciente. Objetivo: Determinar la correlación que existe entre la comunicación asertiva y su relación enfermero- paciente por el uso constante de la mascarilla. Metodología: Enfoque Cuantitativo de diseño descriptivo, observacional analítico de corte transversal, la población fue de 119 personas, se aplicó una encuesta en línea para la obtención de información y fueron analizadas mediante el programa Microsoft Excel y SPSS Estatistics v.22, bajo los principios éticos establecidos en la Declaración de Helsinki. Resultados: La comunicación asertiva y relación enfermeropaciente en cuanto a su correlación se encontró una asociación negativa alta demostrada a través del análisis de Pearson. En cuanto al estilo de comunicación es no asertivo porque no existió claridad al comunicarse con un $85 \%(\mathrm{~N}=17)$ dimensión predominante en los profesionales de enfermería, donde influyó también que no existió confianza entre enfermero, paciente con $75 \%(\mathrm{~N}=76)$ dichos factores se debieron al uso constante de la mascarilla. Conclusiones: Mediante la utilización de la prueba estadística de correlación Pearson que mostró una asociación significativa baja de -0,095 se exteriorizó la dependencia existente entre las dos variables.

Palabras Clave: comunicación asertiva; relación enfermero-paciente; correlación. 


\title{
Assertive communication and its nurse-patient relationship due to the constant use of the face mask
}

\begin{abstract}
Introduction: Assertive communication in our environment is a fundamental process in all social relationship, that regulates and enables interaction with people when providing comprehensive and quality care, by using clear and understandable terminologies, allowing to perform a bond of trust and safety between nurse-patient. Objective: To determine the correlation that exists between assertive communication and the nursepatient relationship due to the constant use of the face mask. Methodology: Quantitative approach of descriptive design, cross-sectional analytical observational, the population was 119 people, an online survey was applied to obtain information and they were analyzed using the Microsoft Excel program and SPSS Statistics v.22, under the ethical principles established in the Declaration of Helsinki. Results: Assertive communication and nurse-patient relationship in terms of their correlation, a high negative association was found, demonstrated through Pearson's analysis. Regarding the communication style, it is not assertive since there was no clarity when communicating with $85 \%(\mathrm{~N}=17)$, the predominant dimension in nursing professionals, which also influenced that there was no trust between nurse-patient with $75 \%(\mathrm{~N}=76)$ these factors were due to the constant use of the mask. Conclusions: Using the Pearson correlation statistical test, which showed a low significant association of $-0,095$, the dependence between the two variables was externalized.
\end{abstract}

Keywords: assertive communication; nurse-patient relationship; correlation.

Artículo recibido: 30 noviembre. 2021 Aceptado para publicación: 29 diciembre 2021 Correspondencia: eyanchapanta5231@uta.edu.ec Conflictos de Interés: Ninguna que declarar 


\section{INTRODUCCIÓN}

Desde el inicio de la enfermería con Florence Nightingale, ya se planteaba la importancia y la necesidad de la comunicación y relación con los pacientes ( Martínez Tovar, A.et al. (2017).Se determina que la comunicación es un arte, una habilidad que debe ser dominada por el personal enfermero/a pues conlleva hacer frente no sólo a los pacientes sino también a sus familias, a los médicos, y al resto del equipo profesional de salud ( Flores-Romo, A. G.et al., (2019).

La comunicación asertiva en el entorno es un elemento principal, es decir; es la interacción entre seres humanos que permiten al individuo establecer una relación con los demás ( Solano, 2017).Hoy por hoy conocemos con certeza que la comunicación se puede aprender, pero que ese aprendizaje está relacionado con las características de las personas que aprenden, y, por lo tanto, sobre la base de una serie de rasgos de personalidad ( Gil, Y. Y. C,2021).Todos tenemos la posibilidad de aprender y mejorar nuestras habilidades de comunicación si seguimos la metodología adecuada.

Por otro lado, podemos señalar que dentro de la relación enfermera-paciente la comunicación es uno de los factores claves a la hora de prestar cuidados integrales y de calidad( Rioboo_valle_veroniatfg.pdf.s. f.). La comunicación cobra aún más valor en los pacientes pues es la mejor manera de acercarnos a la persona para saber de qué manera podemos ayudarle ( Jiménez et al., 2018).En muchas ocasiones este proceso comunicativo tan importante puede verse delegado a un segundo plano por la falta de tiempo debida a la sobrecarga de trabajo, o por la falta de entrenamiento de los profesionales en estos aspectos ( Fuentes y Pujals,2017). Esta situación de la comunicación puede afectar negativamente la calidad de los cuidados y dar como resultado una mala atención a los pacientes.

En la actualidad, hay una serie de problemas que han desencadenado a una deficiencia en la comunicación por el uso constante de la mascarilla que han provocado que la comunicación sea difícil de entender entre el personal de salud y los pacientes, con mayor frecuencia en personas que presentan pérdidas auditivas enfrentan mayores desafíos de comunicación pues la mascarilla reduce el volumen y la claridad del habla ( Benítez, 2021). 
La Asertividad incluye el desarrollo de nuevos modelos mentales que permiten actuar desde un estado interior de confianza y seguridad; en lugar de la emocionalidad limitante típica de la ansiedad, la culpa o el enojo (García Pérez, 2016).

Muchas veces se descubre que aún con las mejores intenciones, aun haciendo lo mejor posible por comunicarnos según lo que hemos aprendido correctamente, no logramos hacerlo asertivamente; de esta manera construimos vínculos y sostenemos conversaciones complejas, desgastantes que generan un impacto negativo en nuestra calidad de vida ( Castanyer Mayer-Spiess, O, 2014).

Según señala Jaramillo (2016), en la actualidad la comunicación asertiva se considera una responsabilidad de los enfermeros y como una herramienta para la humanización del cuidado. La prestación de una atención de calidad no solo requiere una adecuada preparación en cuanto a conocimientos científicos, sino que además es necesario el desarrollo de habilidades comunicativas, que les permiten a los enfermeros enfrentarse a diversas situaciones en su quehacer profesional.

DeVicente (2018), manifiesta que las personas asertivas saben lo que desean, están seguras de sí mismo, y en esa misma medida, tienen un alto nivel de confianza y autoestima, establecen una comunicación clara y directa con los demás, son proactivas buscando continuamente sus objetivos planteados, y aceptan las limitaciones que tanto los elementos como las personas en algún momento pueden interponerles.

La comunicación asertiva abarca también varios tipos ya sea verbal o no verbal, de manera general ( Aguilar, 2019). Para que la comunicación sea asertiva, se deben tomar en cuenta una serie de elementos que la caracterizan y que tienen que ver con aspectos psicológicos, emotivos y pragmáticos del acto comunicativo. Así, por ejemplo: Duración del habla, Tono, voz, Claridad y la velocidad con la que se transmite la información, así como también valoraremos la empatía, confianza y el respeto mutuo (Adell Herrera, J, 2018) (Benavent M, Ferrer E, Francisco del Rey C, 2018).

\section{METODOLOGÍA}

Se realizó una investigación Cuantitativa, de diseño descriptivo, observacional analítico de corte transversal. Pues en esta investigación se pretende abordar nuevos conocimientos y practicas acerca la comunicación asertiva por el uso de la mascarilla desde la perspectiva del personal de enfermería y los pacientes. 
La población estuvo conformada por 119 profesionales de enfermería y pacientes del centro de salud N-1 Ambato, se tomó en cuenta los siguientes criterios de inclusión: enfermero/as asistenciales del centro salud y pacientes que desearon participar voluntariamente en esta investigación. Entre los criterios de exclusión se consideraron al personal de salud que no labora en el lugar de la investigación y aquellos pacientes que tengan algún grado de discapacidad que no puedan contestar la encuesta.

Se utilizó encuestas validadas por Noel Ocampo; Edith Atuncar\& Ramiro Samaniego, lo cual se estructuro por dimensiones y preguntas donde la primera variable fue de comunicación asertiva que constó de 12 ítems con respuestas de siempre con un valor de 3 , a veces con 2 puntos y nunca 1 punto donde se evaluó la claridad, el tono de voz adecuado y la expresión de opinión y sentimientos, y para la segunda variable de relación enfermero-paciente consto de 12 ítems las cuales fueron medidas con bueno (Si),regular (A veces), malo (No) donde se evaluó la empatía, Confianza y Respeto mutuo, estos fueron divididas en aspectos sociodemográficos: como género y edad, de igual manera aspectos socioculturales como: la relación enfermero paciente por el uso de la mascarilla para obtener una comunicación asertiva, la cual fue validada por medio del indicador Alfa de Cronbach, alcanzando un nivel de confiabilidad de 0.79 lo cual determinó al instrumento apto para los propósitos de la investigación (Ocampo N, Vázquez S, 2014) ( Calua Cueva, M. R. Delgado Hernández, Y. L. \& López Regalado, Óscar, 2021).

En lo referente a las consideraciones éticas de los participantes de la investigación su información se encuentra salvaguardada bajo los principios éticos establecidos en la Declaración de Helsinki, los cuales comprometen al investigador a la protección de la dignidad, autonomía, privacidad y confidencialidad de los datos de la población objeto de estudio (Barrios OI, Anido EV, Morera PM, 2016).

En lo concerniente a la aplicación del instrumento se hizo uso de los medios digitales por medio de una encuesta online, se aplicó de manera anónima en la plataforma Microsoft Forms, seguidamente se analizó mediante el programa de Microsoft Excel y SPSS Statistics v.22 con la elaboración de tablas y gráficas con los resultados obtenidos.

\section{RESULTADOS Y DISCUSIÓN}

Del total de 119 encuestados comprendidos entre la edad de 15-65 años de edad, se deduce que 71 profesionales de enfermería y pacientes pertenecen al sexo femenino, 
mientras que 48 son del sexo masculino. El género predominante es el sexo femenino entre profesionales de enfermería y pacientes.

De los encuestados el $16.8 \%$ corresponden al personal de enfermería, y el $83.2 \%$ a los pacientes que asistieron al centro de salud.

Tabla 1. Comunicación Asertiva

\begin{tabular}{|c|c|c|c|c|c|c|c|c|c|c|}
\hline & & Comu & icac & n ase & & & & & & \\
\hline \multirow[b]{2}{*}{ Dimensión } & \multicolumn{2}{|c|}{ Siempre(3) } & \multicolumn{2}{|c|}{$A \operatorname{veces}(2)$} & \multicolumn{2}{|c|}{ Nunca(1) } & \multicolumn{2}{|c|}{ Total } & \multicolumn{2}{|c|}{ Total de categoria } \\
\hline & $\mathbf{N}^{\circ}$ & $\%$ & $\mathbf{N}^{\circ}$ & $\%$ & $\mathbf{N}^{\circ}$ & $\%$ & $\mathbf{N}^{\circ}$ & $\%$ & $\begin{array}{c}\text { Asertivo } \\
\text { 30-36 }\end{array}$ & $\begin{array}{c}\text { No } \\
\text { Asertivo } \\
\text { 12-19 } \\
\end{array}$ \\
\hline \multirow{4}{*}{ Claridad } & 3 & $15 \%$ & 16 & $80 \%$ & 1 & $5 \%$ & 20 & $100 \%$ & 2 & 1 \\
\hline & 5 & $25 \%$ & 4 & $20 \%$ & 11 & $55 \%$ & 20 & $100 \%$ & 1 & 2 \\
\hline & 8 & $40 \%$ & 9 & $45 \%$ & 3 & $15 \%$ & 20 & $100 \%$ & 2 & 1 \\
\hline & 1 & $5 \%$ & 2 & $10 \%$ & 17 & $85 \%$ & 20 & $100 \%$ & 1 & 3 \\
\hline \multirow{4}{*}{$\begin{array}{c}\text { Tono de voz } \\
\text { adecuado }\end{array}$} & 4 & $20 \%$ & 15 & $75 \%$ & 1 & $5 \%$ & 20 & $100 \%$ & 2 & 1 \\
\hline & 4 & $20 \%$ & 3 & $15 \%$ & 13 & $65 \%$ & 20 & $100 \%$ & 1 & 2 \\
\hline & 7 & $35 \%$ & 9 & $45 \%$ & 4 & $20 \%$ & 20 & $100 \%$ & 2 & 1 \\
\hline & 5 & $20 \%$ & 3 & $15 \%$ & 12 & $60 \%$ & 20 & $100 \%$ & 1 & 2 \\
\hline \multirow{4}{*}{$\begin{array}{c}\text { Expresión de } \\
\text { opinión y } \\
\text { sentimientos }\end{array}$} & 4 & $20 \%$ & 2 & $10 \%$ & 14 & $70 \%$ & 20 & $100 \%$ & 1 & 2 \\
\hline & 3 & $15 \%$ & 2 & $10 \%$ & 15 & $75 \%$ & 20 & $100 \%$ & 1 & 2 \\
\hline & 3 & $15 \%$ & 16 & $80 \%$ & 1 & $5 \%$ & 20 & $100 \%$ & 2 & 1 \\
\hline & 4 & $20 \%$ & 14 & $70 \%$ & 2 & $10 \%$ & 20 & $100 \%$ & 2 & 1 \\
\hline & & & & & & & & & 18 & 19 \\
\hline
\end{tabular}

Fuente: Encuesta realizada a los profesionales de Enfermería y pacientes del Centro de Salud N-1 Ambato.

Elaborado por: Erika Yanchapanta

Según los resultados arrojados por la tabla 1 , se indica que $85 \%(\mathrm{~N}=17)$ de los profesionales de enfermería nunca han presentado claridad al comunicarse con los pacientes por el uso constante de la mascarilla lo que les dificulta realizar una comunicación de calidad, mientras que el $80 \%(\mathrm{~N}=16)$ responde que a veces pueden expresar opiniones y sentimientos referente al estado o situación en el que se encuentre el paciente, y el $75 \%(\mathrm{~N}=15)$ indica que los profesionales de enfermería a veces tienen un tono de voz adecuado al momento de comunicarse con el paciente o el personal de 
salud porque no se escucha o simplemente no se entiende lo que el profesional desea decir.

Tabla 2. Relación Enfermero-Paciente

\begin{tabular}{|c|c|c|c|c|c|c|c|c|}
\hline \multicolumn{9}{|c|}{ Relación enfermero-paciente } \\
\hline \multirow[b]{2}{*}{ Dimensión } & \multirow{2}{*}{\multicolumn{2}{|c|}{$\begin{array}{c}\text { Buena) } \\
(\mathrm{Si})\end{array}$}} & \multirow{2}{*}{\multicolumn{2}{|c|}{$\frac{\text { Regular }}{\text { (A veces) }}$}} & \multirow{2}{*}{\multicolumn{2}{|c|}{$\begin{array}{c}\text { Nunca } \\
\text { (no) }\end{array}$}} & \multicolumn{2}{|c|}{ Total } \\
\hline & & & & & & & & \\
\hline \multirow{5}{*}{ Respeto mutuo } & $\mathbf{N}^{\circ}$ & $\%$ & $\mathbf{N}^{\circ}$ & $\%$ & $\mathbf{N}^{\circ}$ & $\%$ & $\mathbf{N}^{\circ}$ & $\%$ \\
\hline & 35 & $35 \%$ & 38 & $38 \%$ & 26 & $26 \%$ & 99 & $100 \%$ \\
\hline & 7 & $7 \%$ & 52 & $52 \%$ & 40 & $40 \%$ & 99 & $100 \%$ \\
\hline & 32 & $50 \%$ & 53 & $54 \%$ & 14 & $14 \%$ & 99 & $100 \%$ \\
\hline & 29 & $29 \%$ & 46 & $46 \%$ & 24 & $24 \%$ & 99 & $100 \%$ \\
\hline \multirow{4}{*}{ Empatía } & 12 & $12 \%$ & 19 & $19 \%$ & 68 & $68 \%$ & 99 & $100 \%$ \\
\hline & 37 & $37 \%$ & 13 & $13 \%$ & 49 & $49 \%$ & 99 & $100 \%$ \\
\hline & 38 & $38 \%$ & 43 & $43 \%$ & 18 & $18 \%$ & 99 & $100 \%$ \\
\hline & 20 & $20 \%$ & 11 & $11 \%$ & 68 & $68 \%$ & 99 & $100 \%$ \\
\hline \multirow{4}{*}{ Confianza } & 11 & $11 \%$ & 16 & $16 \%$ & 72 & $73 \%$ & 99 & $100 \%$ \\
\hline & 30 & $30 \%$ & 9 & $9 \%$ & 60 & $60 \%$ & 99 & $100 \%$ \\
\hline & 20 & $20 \%$ & 17 & $17 \%$ & 62 & $62 \%$ & 99 & $100 \%$ \\
\hline & 13 & $13 \%$ & 10 & $10 \%$ & 76 & $76 \%$ & 99 & $100 \%$ \\
\hline
\end{tabular}

Fuente: Encuesta realizada a los profesionales de Enfermería y pacientes del Centro de Salud N-1 Ambato.

Elaborado por: Erika Yanchapanta

Como se puede evidenciar en la tabla $\mathrm{N}-2$, el $76 \%(\mathrm{~N}=76)$ indica que no existe confianza entre enfermero-paciente pues no presenta en su totalidad la disposición al diálogo y a escuchar, como también no utilizan terminologías claras y comprensibles que faciliten la comunicación y que le permitan al paciente identificar y satisfacer sus necesidades, continuando con el $68 \%(\mathrm{~N}=68)$ responden que no existe empatía de parte de la enfermera porque no perciben los sentimientos, preocupaciones, perspectivas y experiencias del paciente, y el 54\%(N=53) manifiestan que a veces existe respeto mutuo entre enfermero paciente ya sea por la sobrecarga de labores o por el mismo desinterés de comunicación tanto del personal de enfermería o del mismo paciente. 
Gráfico 1. Estilos de Comunicación

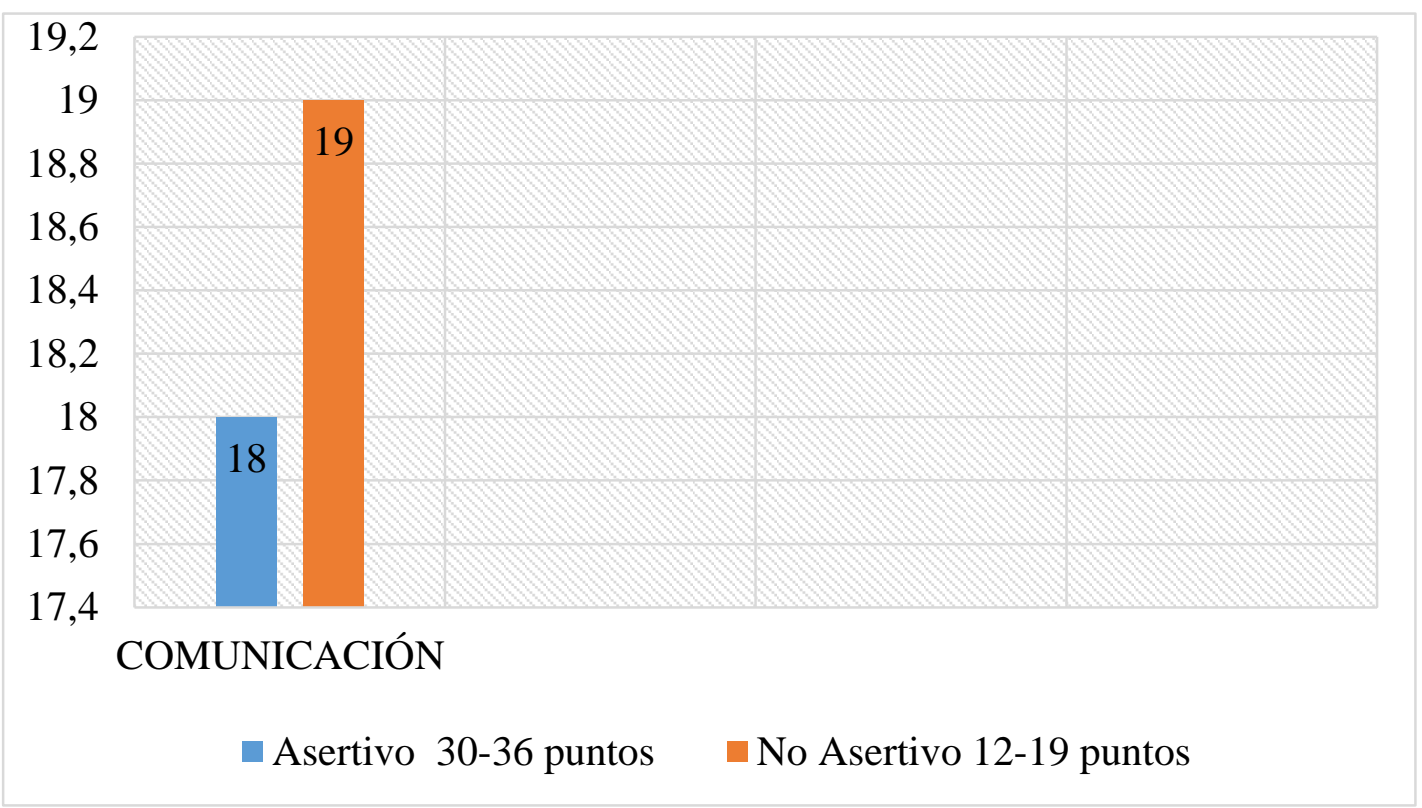

Fuente: Encuesta realizada a los profesionales de Enfermería y pacientes del Centro de Salud N-1 Ambato.

Elaborado por: Erika Yanchapanta

En la gráfica N-1 se observa según los resultados de las variables evaluados según el mayor y menor grado de asertividad que no existe comunicación asertiva entre enfermero-paciente con un valor de 19 puntos que se encuentra dentro del rango 12-19 como lo determinó la categoría final según las dimensiones antes descritas.

Tabla 3. Correlación: Comunicación Asertiva Y Relación Enfermero-paciente

\begin{tabular}{|c|c|c|c|}
\hline \multicolumn{4}{|c|}{ Correlaciones } \\
\hline & & $\begin{array}{l}\text { Comunicación } \\
\text { Asertiva }\end{array}$ & $\begin{array}{c}\text { Relación } \\
\text { Enfermero-Paciente }\end{array}$ \\
\hline \multirow{3}{*}{$\begin{array}{l}\text { Comunicación } \\
\text { Asertiva }\end{array}$} & $\begin{array}{l}\text { Correlación de } \\
\text { Pearson }\end{array}$ & 1 &,- 095 \\
\hline & Sig. (bilateral) & & 691 \\
\hline & $\mathrm{N}$ & 20 & 99 \\
\hline \multirow{3}{*}{$\begin{array}{l}\text { Relación } \\
\text { Enfermero- } \\
\text { Paciente }\end{array}$} & $\begin{array}{l}\text { Correlación de } \\
\text { Pearson }\end{array}$ &,- 095 & 1 \\
\hline & Sig. (bilateral) & ,691 & \\
\hline & $\mathrm{N}$ & 20 & 99 \\
\hline
\end{tabular}

Fuente: Encuesta realizada a los profesionales de Enfermería y pacientes del Centro de Salud N-1 Ambato.

Elaborado por: Erika Yanchapanta 
En la tabla N-3, se pudo evidenciar que no existe una correlación significativa de -,095 entre las dimensiones y categorías de comunicación asertiva y relación enfermeropaciente con una significancia bilateral de 0,69 en el personal de enfermería y los pacientes por el uso constante de la mascarilla.

A través del análisis de datos obtenidos en la investigación, conjuntamente con la interpretación de distintos estudios que se asemejan al mismo, se busca relacionar y por ende llegar a la discusión de resultados pertinente (Mastrapa, Y., \& Gibert Lamadrid, M, 2016).

De la población utilizada en la investigación, se tiene que la mayoría de participantes se encontraron 71 mujeres y 48 hombres entre el personal de enfermería y pacientes del centro de salud comprendidos entre la edad de 15-65 años respectivamente.

De la encuesta aplicada en línea a los profesionales de enfermería y a los pacientes que asistieron al centro de salud N-1 Ambato, se obtuvo que no existe correlación significativa entre las dos variables pues se reflejó con -0,095 valor analizado mediante el análisis de Pearson donde no se vio reflejado su énfasis en la habilidad de relacionarse y de mantener una comunicación significativa con el paciente (Lalinde JDH, Castro FE, Rodríguez JE, 2018) (Allamde Cusso R, Macías Seda J, Porcel Gálvez AM, 2019).

Con respecto al estilo de comunicación resultó ser no asertivo debido a la falta de interés en la comunicación, la no empatía del personal de enfermería (Quiñonez Fuentes, Jennifer Zurina, y Genaro Moyano Arcos, 2019), a diferencia de (Cedeño, 2020) denominado "Técnicas para mejorar la comunicación asertiva de los padres de familia en la unidad educativa físcomisional Mariana de Jesús” quien reportó un tipo de comunicación asertiva mayoritariamente debido a que los profesionales de enfermería tienen la capacidad de adaptarse a grupos e interactuar con los demás, lo que no se evidenció en la investigación realizada, contrariamente a lo encontrado por Valderrama(2018) en la ciudad de Trujillo-Perú realizaron una investigación titulada

\section{"Comunicación asertiva y desempeño laboral de la enfermera. Hospital Leoncio}

Prado de Huamachuco" quien reportó un alto nivel de comunicación asertiva tanto del personal de enfermería como del mismo paciente debido a que la comunicación que emplearon fueron gestos, palabras y todo de voz adecuado en el campo laboral, la misma que logro llevar a una buena relación enfermero- paciente. Al respecto que el 
personal de enfermería a veces presente un tono de voz adecuado, se debería a la manera agresiva o en muchos de los casos a los gritos al momento de comunicarse que se asemeja a los criterios ejecutados por Sandoval (2018) en Ecuador denominado "La comunicación del talento humano en la productividad empresarial" quien reportó que el tono de voz aplicarse era inadecuado debido a la tonalidad al hablar y a su postura.

Con respecto a las profesiones de enfermería no existe empatía ni confianza pues no comprenden la realidad, los sentimientos y las emociones de los pacientes como también nos les proporcionan predisposición para que exista ese laso de confianza, criterio que concuerda con la de Triana M, en el año(2017) llamado "La empatía en la relación enfermero-paciente y a la de Vargas et al, en (2020) en la investigación titulada "La confianza en la relación profesional de la salud y paciente" donde la confianza y la empatía eran bajos, se debió al tipo de establecimiento donde fue realizada la investigación, donde el profesional de enfermería disponía de un tiempo mínimo para dedicarse a la interacción con el paciente y por la sobrecarga de labores.

Mediante este artículo se contribuye en el aporte significativo de información, haciendo hincapié en la aportación de datos fundamentales que se visualizaron por fuentes reales de investigación científica, realizadas al personal de enfermería y a los pacientes para llevar acabo las experiencias que presentan a diario en el centro de salud.

\section{CONCLUSIÓN O CONSIDERACIONES FINALES}

Tras el proceso de investigación realizado, se concluyó mediante el análisis de Pearson, no existe correlación significativa entre comunicación asertiva y la relación enfermeropaciente, el cual muestra una asociación de -0,095 el cual manifiesta a través de esta experiencia la dependencia existente entre los dos objetos de estudio.

Se deduce que el estilo de comunicación utilizado en la actualidad es no asertivo debido a varios factores como: en el que el enfermero no expresa la información con claridad debido a que no emplea un tono de voz adecuado por el uso constante de la mascarilla, por ello al paciente no le permite expresar con confianza sus emociones, sentimientos y perspectivas acerca su estado de salud.

En cuanto al uso constante de la mascarilla presentó una influencia negativa en la comunicación y relación enfermero-paciente, donde se determinó que el uso permanente de la misma, no permite una expresión clarificada por la persona, por otro lado, el 
mismo estrés generalizado por la situación de la pandemia Covid-19, porque la mascarilla es de uso necesario para el cuidado y protección de la salud.

\section{LISTA DE REFERENCIA}

Martínez Tovar, A., Rojas Cartillo, X., Rosas Vargas, L., Zárate Grajales, R. A., \& Flores Mora, L. F. (2017). FLORENCE NIGHTINGALE, PIONERA EN EL MODELO DE GESTIÓN.RevistaCuidArte,6(11),44. https://doi.org/10.22201/fesi.23958979e.2017.6.11.69136

Flores-Romo, A. G., Zorrilla-Martínez, L. B., Trejo-Ortiz, P. M., Araujo-Espino, R., \& Calderón-Ibarra, A. (2019). Características de la comunicación enfermeropaciente en un hospital de segundo nivel de Zacatecas. Enfermería. Innovación y Ciencia, 1(1), 9-9.

Solano (2017). COMUNICACIÓN ASERTIVA ANALISIS BIBLIOGRAFICO DE

L.pdf. (s.f.). Recuperado 15 de noviembre de 2021, de https://repository.udistrital.edu.co/bitstream/handle/11349/12970/Guti\%c3\%a9rr ezSolanoLauraFernanda2018.pdf?sequence $=1 \&$ is Allowed $=\mathrm{y}$

Gil, Y. Y. C. (2021). El cine: Herramienta pedagógica para la comunicación asertiva en la formación integral del ser humano. 31.

Rioboo_valle_veroniatfg.pdf. (s. f.). Recuperado 15 de noviembre de 2021, de https://repositorio.uam.es/bitstream/handle/10486/685141/rioboo_valle_veroniat fg.pdf?sequence $=1 \&$ isAllowed $=y$

Jiménez et al. - Módulo 1- Técnicas y estrategias de comunicación p.pdf. (s. f.). Recuperado 15 de noviembre de 2021, de http://www.comsegovia.com/pdf/cursos/2018/Modulo\%201\%20La\%20comunic acion.pdf

Fuentes y Pujals. MODELO DE COMUNICACIÓN PARA LA ENSEÑANZA A DISTANC.pdf. (s.f.). Recuperado 15 de noviembre de 2021, de https://www.tdx.cat/bitstream/handle/10803/4126/caf1de2.pdf

Benítez. MAGÍSTER EN GESTIÓN DE PERSONAS Y DINÁMICA ORGANIZ.pdf. (s.f.). Recuperado 15 de noviembre de 2021, de https://repositorio.uchile.cl/bitstream/handle/2250/178909/Tesis\%20$\% 20$ Arlette\%20Leup\%c3\%adn.pdf?sequence=1\&isAllowed=y 
García Pérez (2016). Mejora tu calidad de vida y rendimiento académico .pdf. (s. f.). Recuperado 15 de noviembre de 2021, de https://web.archive.org/web/20180702092801id_/https://www.zaragoza.unam.m x/portal/wp-content/Portal2015/publicaciones/libros/Mejora-calidad-vidarendimientoacademico-autoaprendizaje.pdf

Castanyer Mayer-Spiess, O. (2014). Aplicaciones del asertividad. Bilbao, Editorial Desclée de Brouwer. Recuperado de: https://elibro.net/es/lc/uta/titulos/115847. Jaramillo, (2016). Comunicación asertiva, una habilidad clave en enfermería desde la teoría de Hildegard Peplau. Universidad de la Sabana Chía - Cundinamarca. Medellí. Colombia.

DeVicente, B. (2018). psicologoonlinedevicente.com. Obtenido de Psicólogo Online de Vicente. Recuperado 15 de noviembre de 2021 de http://www.psicologoonlinedevicente.com/asertividad-y-empatia/

Aguilar. COMUNICACIÓN ASERTIVA.pdf. (s. f.). Recuperado 15 de noviembre de 2021, de http://www.direcciondepersonal.com/comunicacion_asertiva.pdf

Adell Herrera, J. (2018). ¿Hablar o comunicar? Barcelona, Editorial UOC. Recuperado de https://elibro.net/es/lc/uta/titulos/116298.

Benavent M, Ferrer E, Francisco del Rey C. Fundamentos de Enfermería. En: Colección de Enfermería 21. (2018). Difusión Avances de Enfermería Madrid-España Recuperadodefile:///D:/Users/SILVIO/Downloads/DialnetLaComunicacionPieza ClaveEnEnfermeria-4069152.pdf

Ocampo N, Vázquez S (2014) Método de comunicación asertiva. 2ra ed; Edit. Trillas México.p.35-36.

Atuncar_tej.pdf. Recuperado 15 de noviembre de 2021 de: https://repositorio.usmp.edu.pe/bitstream/handle/20.500.12727/6490/atuncar_tej. pdf? sequence $=1 \&$ is Allowed

Colino S. Uso de mascarillas impide la comunicación AARP. recuperado 15 de noviembre de 2021 de: https://www.aarp.org/espanol/salud/enfermedades-ytratamientos/info

Calua Cueva, M. R. Delgado Hernández, Y. L. \& López Regalado, Óscar (2021). Comunicación asertiva en e 1 contexto educativo: revisión sistemática. Revista Boletín Redipe, 10(4), 315-334. https://doi.org/10.36260/rbr.v10i4.1274 
Barrios OI, Anido EV, Morera PM (2016) Declaración de Helsinki: cambios y exégesis. Revista Cubana de Salud Pública.;42(1):132-142.

Mastrapa, Y., \& Gibert Lamadrid, M. (2016). Relación enfermera-paciente: una perspectiva desde las teorías de las relaciones interpersonales. Revista Cubana de

Enfermería,32(4).Recuperadode http://www.revenfermeria.sld.cu/index.php/enf/ article/view/976

Lalinde JDH, Castro FE, Rodríguez JE (2018). Sobre el uso adecuado del coeficiente de correlación de Pearson: definición, propiedades y suposiciones. Pg 37:16.

Allamde Cusso R, Macías Seda J, Porcel Gálvez AM (2019). La relación enfermerapaciente: identidad histórica, metodológica y terapéutica en los cuidados de enfermería. Cult Los Cuid.;23(55):78.

Quiñonez Fuentes, Jennifer Zurina, y Genaro Moyano Arcos. (2019). La asertividad como estilo de comunicación en la formación del sujeto educador». Revista Scientific 4(Ed. Esp.):68-83. doi: 10.29394/Scientific.issn.2542-2987.2019.4. E.4.68-83.

Cedeño, Fanny Mirella Herrera. (2020). Técnicas para mejorar la comunicación asertiva de los padres de familia, de la unidad educativa fiscomisional "Mariana de Jesús" del cantón El Tambo, provincia del Cañar. (Revisión). Roca. Revista científico - educacional de la provincia Granma 16:824-35.

Valderrama, Bach Enf Arana, Jhuliza Yubicxa, Bach Enf Aranda Palomino, y Mariela Rosario. s.f. «TESIS PARA OPTAR EL TÍTULO PROFESIONAL DE: LICENCIADA EN ENFERMERÍA». Pg. 81.

Sandoval Ramos, Segundo José, David Alejandro Armijos Romero, y Karen Gabriela González Caraguay. 2018. «La comunicación del talento humano en la productividad empresarial». INNOVA Research Journal 3(8.1):167-75. doi: 10.33890/innova. v3.n8.1.2018.760.

Puig, Inma. (2019). La revolución emocional: ¡Todos necesitamos lo mismo para sentirnos bien! Penguin Random House Grupo Editorial España.

Triana-Restrepo, Martha Cecilia. (2017). La empatía en la relación enfermerapaciente». Avances en Enfermería 35(2):120. doi: 10.15446/av.enferm.v35n2.66941. 
Vargas Celis, I., Soto Guerrero, S., Hernández Leal, M., \& Campos Romero, S. (2020). La confianza en la relación profesional de la salud y paciente. Revista Cubana de Salud Pública, 46(3). Recuperado de http://www.revsaludpublica.sld.cu/index.php/spu/article/view/1575 